organizers of astronomy seem to have lost sight completely of the fact that scientifically the whole purpose of observation is to provide a basis for theory. Despite its being the subject of such as Newton, Gauss, Laplace, Lagrange, and Euler, theoretical astronomy has been cast to the tender mercies of private enterprise, but without financial support, and as a result the situation is that obscurant speculations can gain acceptance more readily than genuine theoretical work--it is all lumped together by most of our observational astronomers as 'mere theory'--because in failing to accord with scientific method such speculations appeal to the majority on their own philosophical level.

Everything that the skill of printer and publisher can provide has been lavished on this book, which from a production point of view is quite excellent, and it is sad that much of the content falls so far below that level. One must express the hope that the University of Chicago Press will take early steps to have it thoroughly revised.

R. A. LytetLeton

\section{MATHEMATICS AND WEATHER}

An Introduction to the Hydrodynamical Methods of Short Period Weather Forecasting

By I. A. Kibel. Translation edited by R. Baker. Pp. xiii +383 . (Oxford, London, New York and Paris: Pergamon Press, 1963.) 100s.

A TTEMPTS to use the differential equations of hydrodynamics and thermodynamics to predict the changes of atmospheric pressure had to await the development of high-speed computers because the equations are so complicated that only arithmetic methods of solution are possible; the remarkable effort of L. F. Richardson, who carried out a partial solution by hand computation, is alone in preceding the electronic computer. In tho past two decades, research has procooded quickly in many countries and the solution of the equations in simplified form has been one of the major items of meteorological research. Dr. Kibel has been the leader and inspirer of the Russian effort in this field and is highly qualified to write about the recent advances; his book is welcome because there are very few adequate accounts of this branch of meteorology and applied mathematics.

The book, developed from a series of university lectures, gives a logical development typical of applied mathematics text-books. First there is a careful development of the equations of motion of the atmosphere, allowing for thermodynamic effects, and of the mathematical representation of the boundary layer. Next comes the vital chapter dealing with the scale of the atmospheric motions that are to be treated, the relative magnitudes of the terms in the equations when applied to this scale and the simplifications that follow. So far the ideas developed in the U.S.S.R. were similar to those developed elsewhere. The remainder of the book is devoted to the solution of the differential equations in successively more complox cases, starting with the solution for linearized equations, treating the atmosphere like an incompressible fluid. This is followed by a treatment of the linearized threedimensional equations and illustrative examples are given. The corresponding non-linear problems are very carefully treated and really form the core of the book so far as meteorologists are concerned. In solving tho equations the Russians have used the classical methods of constructing Green's functions and so expressing the solution as triple integrals: Dr. Kibel naturally gives prominence to this method of solution, working it out in detail, but he does not neglect to mention the straightforward arithmetical approach which has been favoured in Britain and the United States. (In fact throughout the text it is clear that the author was well aware of the research going on outside his own country.) Of course, when either method of solution is used to obtain practical answers there is an enormous amount of arithmetic computation to be done; on one hand, the Green's integral has to be evaluated at a great number of points as a sum, while, on the other, iterative processes have to be used. It is by no means clear which is the more accurate way of solving the equations or which is the more economic in computing time and it would be interesting to have a comparison made.

Dr. Kibel illustrates the different methods of solving the equations by examples of predictions made both by Soviet and other research workers and also in some cases gives statistical measures which indicate that numerical weather prediction is likely to be a success.

This is a very useful text-book and gives the Russian point of view up to 1956 very clearly and carefully. Much work has been done, both inside and outside Russia, since this text was first published, and it is now by no means an up-to-date account of the state of the art. Nevertheless, its value as a text-book and as an introduction to Russian ideas, so often masked by the language difficulty, remains high and it is probably the most complete account of the subject available in book form. The translation has a number of irritating errors, as has the mathematics. The original text-book had no index and this defect has been remedied in the translation. There are but fow references and no standard method of annotating them; in some cases it would be difficult to trace the original paper. However, these minor deficiencies do not detract from the value of the book.

\section{E. KNIGHTING}

\section{ISOTOPES IN CLINICAL MEDICINE}

The Body Cell Mass and Its Supporting Environment Body Composition in Health and Disease. By Francis D. Moore, Knud H. Olesen, James D. McMurrey, H. Victor Parker, Margaret R. Ball and Caryl Magnus Boyden. Pp. xxv +535. (Philadelphia and London: W. B. Saunders Company, 1963.) $161 s$.

7 HE investigation of body composition represents one of the most favourable fields of application of isotopes and owes much to Georg von Hevesy, to whom this volume is dedicated. Accuracy and convenience of isotopic measurements are now such that in spite of unavoidable restrictions which apply to the use of radioactive materials in patients the hospital test laboratory of the future can confidently bo visualized as a major centre of isotopic activities, and many books similar in genoral character to the present volume may be anticipated. This one describes 10 years up to 1954 spent in establishing basic techniques and a subsequent and more intensive similar period of clinical application of these methods at the Peter Bent Brigham and Massachusetts General Hospitals.

Part 1 deals with methods, calculations and interpretations relating to measurements of total-body water and electrolyte masses using a variety of labelled substances. Then comes a consideration of more difficult problems of sub-dividing these between body compartments, sometimes on an anatomical basis, such as into intra- and extravascular or intra- and extra-cellular, but often on a purely functional basis, for example, exchangoable and nonexchangeable phosphorus. In Part 2 the emphasis is on specific clinical conditions including burns, fracture, hæmorrhage, heart disease, renal and hepatic failure, and obesity. It is evident throughout that a now and extremely informative phase is developing in clinical medicine which is likely to have revolutionary effects. 